\title{
Impact of combination antiretroviral therapy initiation on adherence to antituberculosis treatment
}

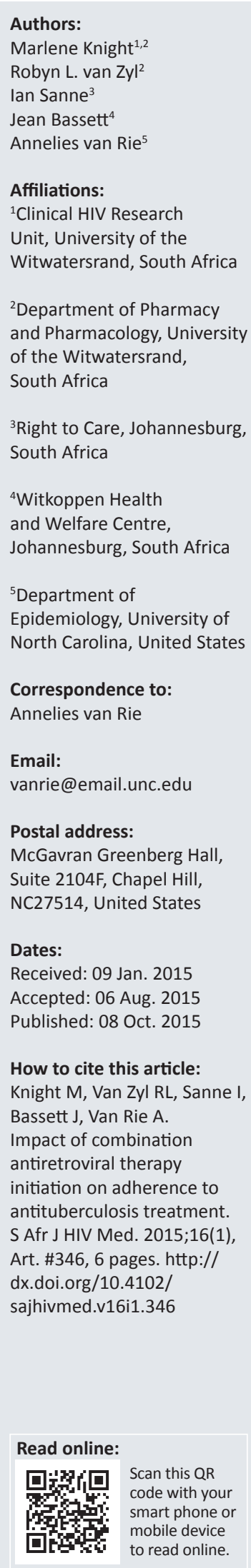

Background: Healthcare workers are often reluctant to start combination antiretroviral therapy (ART) in patients receiving tuberculosis (TB) treatment because of the fear of high pill burden, immune reconstitution inflammatory syndrome, and side-effects.

Object: To quantify changes in adherence to tuberculosis treatment following ART initiation.

Design: A prospective observational cohort study of ART-naïve individuals with baseline CD4 count between 50 cells $/ \mathrm{mm}^{3}$ and 350 cells $/ \mathrm{mm}^{3}$ at start of TB treatment at a primary care clinic in Johannesburg, South Africa. Adherence to TB treatment was measured by pill count, self-report, and electronic Medication Event Monitoring System (eMEMS) before and after initiation of ART.

Results: ART tended to negatively affect adherence to TB treatment, with an $8 \%-10 \%$ decrease in the proportion of patients adherent according to pill count and an $18 \%-22 \%$ decrease in the proportion of patients adherent according to eMEMS in the first month following ART initiation, independent of the cut-off used to define adherence $(90 \%, 95 \%$ or $100 \%)$. Reasons for non-adherence were multifactorial, and employment was the only predictor for optimal adherence (adjusted odds ratio 4.11, 95\% confidence interval 1.06-16.0).

Conclusion: Adherence support in the period immediately following ART initiation could optimise treatment outcomes for people living with TB and HIV.

\section{Introduction}

Adhering to a lengthy course of medication is difficult and poses a challenge to achieving health in people with chronic diseases. Poor adherence to treatment for infectious disease poses a risk to both the individual and community as it can lead to prolonged infectiousness, development of drug resistance, and poor treatment outcomes. Tuberculosis (TB) and the human immunodeficiency virus (HIV) present particular challenges as both are chronic diseases that mainly affect disadvantaged populations and involve complex treatment regimens with potentially severe side-effects. ${ }^{1}$ Treatment adherence for TB and HIV is also affected by beliefs about the origins and transmission of TB and HIV, which can result in stigmatisation of those affected. ${ }^{2}$

The 2012 World Health Organization (WHO) and 2015 South African antiretroviral therapy (ART) guidelines recommend initiating ART in people with TB as soon as possible, within the first 2 weeks of initiating TB treatment for those with profound immunosuppression (CD4 counts $<50$ cells $/ \mathrm{mm}^{3}$ ) and within the first 8 weeks of treatment in all TB patients. ${ }^{3}$ Whilst initiating ART greatly improves the survival and quality of life of TB patients living with $\mathrm{HIV}^{4}$ it also poses challenges to patients and healthcare workers. ${ }^{5}$ Early initiation of ART can result in clinical deterioration related to immune reconstitution inflammatory syndrome (IRIS), toxic effects of drugs, or drug interactions, and increased pill burden. The high pill burden of four anti-TB drugs, antiretroviral drugs, and antimicrobial prophylaxis (cotrimoxazole and fluconazole) against opportunistic infections, as well as possible drug interactions and toxic effects, may jeopardise the patient's adherence to treatment. ${ }^{6}$ As a result, healthcare workers are often reluctant to start ART in patients receiving TB treatment. In 2012, only 57\% of TB patients with HIV were started on $\mathrm{ART},{ }^{7}$ and in the latter group, initiation of ART was often delayed. ${ }^{8}$

In the present study, we aimed to quantify changes in adherence to TB treatment associated with initiation of ART by prospectively measuring adherence to TB drugs immediately before and after initiation of ART. 


\section{Methods \\ Study setting and population}

The study took place at Witkoppen Health and Welfare Centre, a primary care clinic in Johannesburg, South Africa. Adults ( $>18$ years old) diagnosed with pulmonary TB who were ART-naïve at the time of initiation of TB treatment and had a CD 4 count between 50 cells $/ \mathrm{mm}^{3}$ and 350 cells / $\mathrm{mm}^{3}$ were eligible for enrolment. Those with rifampicinresistant TB (defined by Xpert MTB/RIF or culture-based drug susceptibility testing) were excluded as they are referred for care. Those with CD4 counts $<50$ cells $/ \mathrm{mm}^{3}$ were excluded because ART should be initiated as an emergency, limiting the possibility of reliably establishing the level of adherence to TB treatment before ART initiation. Those with CD4 counts $>350$ cells $/ \mathrm{mm}^{3}$ were excluded as these individuals were not eligible for ART, according to the 2010 South African Guidelines, which were current at the time of the study.

All care provision for TB and HIV, including the decision on timing of ART initiation, was performed by the routine clinic staff, without any input from study staff.

\section{Study procedures}

Eligible patients who signed informed consent completed a questionnaire collecting information on socio-demographic information, occurrence of side-effects, and adherence support. Medical files were reviewed to collect details of weight, height, results of TB diagnostics (Xpert MTB/RIF, smear microscopy and culture), TB treatment outcome, ART regimen and start date, and CD4 count and viral load (VL) at baseline and during the first 6-12 months of ART.

Ethical approval was obtained from the Institutional Review Board of the University of North Carolina (10-2317) and the University of the Witwatersrand's Human Research Ethics Committee (M10925).

\section{Adherence measures}

Participants were prospectively monitored for adherence, using pill count (primary measure of adherence), self-report, and an electronic Medication Event Monitoring System (eMEMS). Participants received their TB medication in a Securitainer fitted with an eMEMS lid (eMuM, GeoMed, Stellenbosch, South Africa). Participants were seen by study staff at each clinic visit; the number of visits varied and was determined by the routine clinic care provider. At each visit, the date of visit, number of pills distributed for TB treatment, and prescribed dosage were recorded. At each return visit, the number of pills remaining in the container was recorded, the eMEMS lid was connected to a computer to download data pertaining to when the Securitainer was opened, and the patient was asked, 'In the last week, have you missed any of your doses?' Participants with suboptimal adherence (according to self-report, pill count or eMEMS) were asked the reasons for non-adherence.
To reduce the effect of factors other than ART initiation on adherence to TB treatment, the primary outcome measure was adherence during the 28 days before and 28 days after ART initiation. The number of days included varied by participant because ART could be initiated sooner than 28 days after starting TB treatment, and the number of days between visits was not always exactly 28 days.

Pill count was used to calculate several adherence measures: percentage of the prescribed doses taken (100 $\times$ [number of pills dispensed minus the number of pills returned]/[number of days between clinic visits] $\times$ [daily dose]) and three binary indicators: whether adherence equalled $100 \%, \geq 95 \%$ and $\geq 90 \%$. eMEMS data were used to calculate additional measures of adherence: percentage of the prescribed doses taken $(100 \times$ number of days with bottle openings/number of days between clinic visits) and three binary indicators: whether adherence was 100\%, $\geq 95 \%$ and $\geq 90 \%$. The number of daily bottle openings were truncated to one to avoid overestimating adherence. Data from follow-up visit questionnaires were used to calculate the self-reported adherence $(100 \times[1$ minus the number of missed doses]/number of days between clinic visits) and three binary indicators: whether adherence was $100 \%$, $\geq 95 \%$ and $\geq 90 \%$.

\section{Statistical analysis}

Participant characteristics are presented as absolute and relative frequencies for categorical variables and as medians and interquartile ranges (IQR) for continuous variables.

The effect of ART initiation on adherence to TB treatment was evaluated in two different ways. Firstly, the median change in percentage of prescribed doses of TB drugs taken before and after ART initiation was compared for each of the three continuous adherence measures (pill count, eMEMS and selfreport) using the Wilcoxon matched-pair signed ranks test. Secondly, using the exact McNemar test for paired samples, the proportion of participants adherent to $\mathrm{TB}$ treatment before and after ART initiation was compared for all three binary adherence measures for each of the three methods (pill count, eMEMS and self-report).

To determine factors predictive of optimal adherence (100\% adherence) in the first month after ART initiation, we first performed bivariate analysis to estimate crude odds ratios (OR) and 95\% confidence intervals (CI). We subsequently ran a saturated logistic model containing all selected covariates to estimate adjusted ORs (aOR), and used stepwise backwards elimination to generate a final (reduced) predictive model. We conducted a sensitivity analysis to explore the effect of broadening the definition of optimal adherence to $\geq 95 \%$ and $\geq 90 \%$ adherence post ART. Crude ORs and aORs are presented with standard Wald 95\% CI. All analyses were conducted using STATA 12.1 (Texas, USA). 


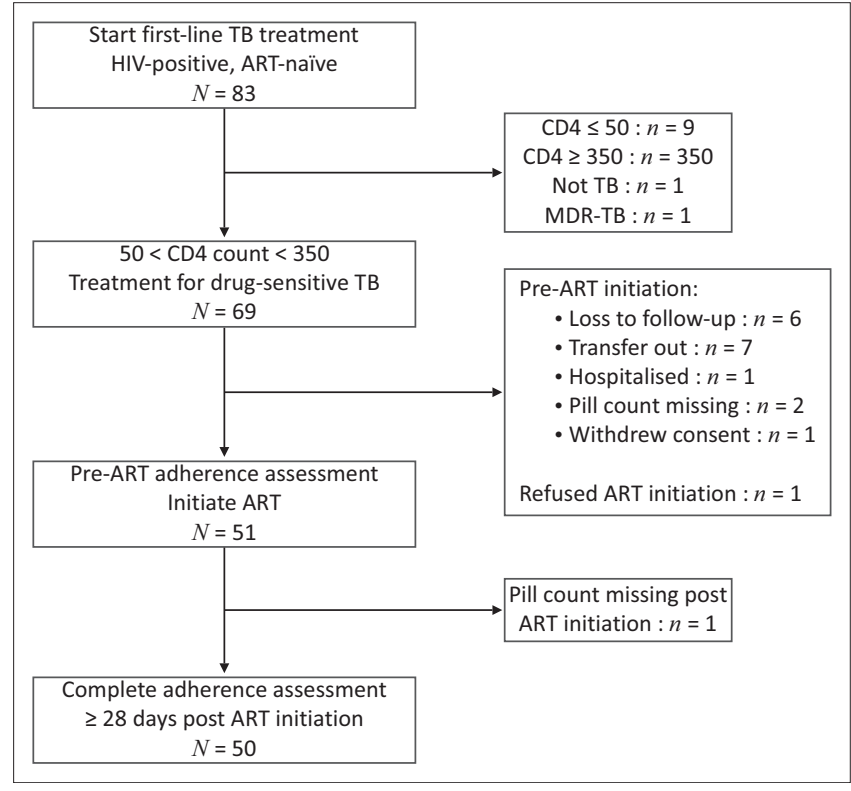

TB, tuberculosis; ART, antiretroviral treatment.

FIGURE 1: Study flow chart.

\section{Results}

\section{Study cohort characteristics}

Between September 2011 and October 2012, 83 ART-naïve individuals initiating first-line drugs for pulmonary TB gave informed consent for study participation (Figure 1). Of these, 14 were excluded from the analysis for not meeting all inclusion criteria: CD4 count $<50$ cells $/ \mathrm{mm}^{3}(n=9)$, CD4 count $>350$ cells $/ \mathrm{mm}^{3}(n=3)$, erroneous TB diagnosis $(n=1)$, or MDR-TB diagnosis $(n=1)$. Prior to ART initiation, another 18 were excluded owing to loss to follow-up ( $n=$ $6)$, transfer to another facility $(n=7)$, hospitalisation $(n=1)$, refusal to start ART $(n=1)$, missing pill count $(n=2)$, and withdrawal of consent $(n=1)$. One additional participant was excluded because of missing pill count post ART initiation.

Among the 50 patients included in the analysis, median age was 32.5 years (IQR 30-38), 56\% were female, 58\% were unemployed, and almost half $(48 \%)$ were not of South African nationality. The diagnosis of TB was bacteriologically confirmed in 80\%. Median CD4 count was 124 (IQR 94-193). The median time to ART initiation was 27 days (IQR 13-48), and almost all (92\%) participants initiated an ART regimen containing efavirenz, lamivudine and tenofovir. TB treatment outcome was successful in $82 \%$. Only $56 \%$ of participants achieved viral load suppression, defined as a viral load $<400$ copies $/ \mathrm{mL}$ in the 6-12 months of ART (Table 1).

\section{Adherence to TB treatment before and after antiretroviral therapy initiation}

When measured by pill count or self-report, the median percentage of prescribed TB drug doses taken was 100\% before and after ART initiation (Table 2). When measured by eMEMS, median percentage of prescribed TB drug doses taken was $93 \%$ before, and $82.5 \%$ (IQR 55-96) after, ART
TABLE 1: Characteristics of 50 individuals included in the analysis of the impact of initiation of antiretroviral treatment on adherence to tuberculosis treatment.

\begin{tabular}{|c|c|c|c|}
\hline Continuous variables & Group & Median $n$ & IQR \% \\
\hline \multirow[t]{2}{*}{ Gender } & Male & 22 & 44.0 \\
\hline & Female & 28 & 56.0 \\
\hline \multirow[t]{2}{*}{ Employed } & Yes & 21 & 42.0 \\
\hline & No & 29 & 58.0 \\
\hline \multirow[t]{3}{*}{ Nationality } & South Africa & 26 & 52.0 \\
\hline & Zimbabwe & 21 & 42.0 \\
\hline & Mozambique & 3 & 6.0 \\
\hline \multirow[t]{4}{*}{ Education $\dagger$} & None & 2 & 4.1 \\
\hline & Primary school & 4 & 8.2 \\
\hline & Some secondary school & 28 & 57.1 \\
\hline & Secondary school completed & 15 & 30.6 \\
\hline \multirow[t]{2}{*}{ TB diagnosis } & Confirmed & 40 & 80.0 \\
\hline & Clinical & 10 & 20.0 \\
\hline \multirow[t]{3}{*}{ ART regimen } & EFV, 3TC, TDF & 46 & 92.0 \\
\hline & EFV, 3TC, D4T & 3 & 6.0 \\
\hline & EFV, 3TC, AZT & 1 & 2.0 \\
\hline \multirow[t]{6}{*}{ TB treatment outcome } & Cure & 9 & 18.0 \\
\hline & Treatment completed & 32 & 64.0 \\
\hline & Treatment failed & 0 & 0.0 \\
\hline & Died & 1 & 2.0 \\
\hline & Lost to follow-up & 5 & 10.0 \\
\hline & Not evaluated (transfer out) & 3 & 6.0 \\
\hline \multirow[t]{5}{*}{ ART outcome } & Suppressed $\$$ & 28 & 56.0 \\
\hline & Failure to suppress & 10 & 20.0 \\
\hline & Died & 1 & 2.0 \\
\hline & Lost to follow-up & 8 & 16.0 \\
\hline & Not evaluated (transfer out) & 3 & 6.0 \\
\hline \multicolumn{2}{|l|}{ Age (years) } & 32.5 & $30-38$ \\
\hline \multicolumn{2}{|l|}{ BMI at enrollment } & 21.5 & $19-23$ \\
\hline \multicolumn{2}{|c|}{ CD4 count at enrollment (cells $/ \mathrm{mm}^{3}$ ) } & 124 & 94-193 \\
\hline \multicolumn{2}{|c|}{ Time from TB treatment to ART initiation (days) } & 27 & $13-48$ \\
\hline
\end{tabular}

$\mathrm{BMI}$, body mass index; TB, tuberculosis; ART, antiretroviral treatment; IQR, interquartile ranges.

$\downarrow$, Education level missing for 1 participant; $\$$, suppression is defined as viral load $<400$ copies $/ \mathrm{mL}$ within the first year of ART.

initiation. There was no change in the percentage of TB medication taken before and after ART initiation, with a median percentage change of $0(\mathrm{IQR}-4,+1)$ for pill count, 0 (IQR 0-0) for self-report, and minus $1.5(\mathrm{IQR}-25,+3)$ for eMEMS (all $p$ values $>0.30$ ).

When measured by pill count, the proportion of participants who were $100 \%$ adherent to TB treatment before and after ART initiation was $64 \%$ (41.7-70.3) versus 56.0\% (50.2-77.8); increased to $68.0(54.6-81.4)$ versus $78 \%(66.1-89.9)$ when adherence was defined as $95 \%$ of prescribed doses taken, and 78.0 (66.1-89.9) versus $88.0 \%$ (78.7-97.3) when adherence was defined as $90 \%$ of doses taken.

Self-reported adherence was high, with $100 \%$ of participants being adherent before and after ART initiation when adherence was defined as taking $\geq 90$ or $\geq 95 \%$ of prescribed doses, and 96.0\% (90.4-100) and 94.0\% (87.2-100) being adherent before and after ART initiation, respectively, when adherence was defined as $100 \%$ of prescribes doses.

Owing to technical errors, power failures, equipment failures, and misunderstandings by pharmacists and patients, adherence data by eMEMS were only available 
TABLE 2a: Adherence to tuberculosis treatment before and after initiation of antiretroviral treatment.

\begin{tabular}{|c|c|c|c|c|c|c|c|c|c|c|}
\hline \multirow[t]{2}{*}{ Adherence measure } & \multicolumn{3}{|c|}{$\begin{array}{l}\% \text { TB drugs prescribed taken before } \\
\text { ART initiation } \dagger\end{array}$} & \multicolumn{3}{|c|}{$\begin{array}{c}\% \text { TB drugs prescribed taken after ART } \\
\text { initiation: }\end{array}$} & \multicolumn{4}{|c|}{$\begin{array}{c}\% \text { change in TB drugs taken before and after ART } \\
\text { initiation }\end{array}$} \\
\hline & $N$ & Median & IQR & $N$ & Median & IQR & $N$ & Median & IQR & $p$ value $\S$ \\
\hline Pill count & 50 & 100 & 96,100 & 50 & 100 & 91,100 & 50 & 0 & $-4,+1$ & 0.55 \\
\hline Electronic MEMS & 21 & 93 & 75,100 & 20 & 82.5 & 55,96 & 14 & -1.5 & $-25,+3$ & 0.31 \\
\hline
\end{tabular}

MEMS, Medication Event Monitoring System; TB, tuberculosis; ART, antiretroviral treatment; IQR, interquartile ranges.

$\dagger$, Period assessed is the period up to 28 days pre ART initiation; $¥$, Period assessed is the 28 -day period following ART initiation. If the first TB clinic visit occurred $>28$ days following ART initiation, the entire time period between ART initiation and subsequent TB clinic visit was included; $\S$, Comparison only possible between 14 patients who had MEMS data available both before and after ART initiation.

TABLE 2b: Hundred percent adherence to tuberculosis treatment before and after initiation of antiretroviral treatment.

\begin{tabular}{|c|c|c|c|c|c|c|c|c|}
\hline \multirow[t]{2}{*}{ Adherence measure } & \multicolumn{3}{|c|}{$\begin{array}{c}\text { Proportion of patients with } 100 \% \\
\text { adherence pre ART }\end{array}$} & \multicolumn{3}{|c|}{$\begin{array}{c}\text { Proportion of patients with } 100 \% \\
\text { adherence post ART }\end{array}$} & \multirow[t]{2}{*}{$\begin{array}{c}\text { Difference in proportion adherent post } \\
\text { v. pre cART (\%) }\end{array}$} & \multirow[t]{2}{*}{$p$ value: } \\
\hline & $N$ & $\%$ & $95 \% \mathrm{Cl}$ & $N$ & $\%$ & $95 \% \mathrm{Cl}$ & & \\
\hline Pill count & 50 & 64.0 & $50.2-77.8$ & 0.50 & 56.0 & $41.7-70.3$ & -8.0 & 0.50 \\
\hline Electronic MEMS & 21 & 38.1 & $15.4-60.7$ & $0.5 \dagger$ & 10.0 & $0.4-24.4$ & -18.1 & $0.5 \dagger$ \\
\hline
\end{tabular}

MEMS, Medication Event Monitoring System; ART, antiretroviral treatment; $\mathrm{Cl}$, confidence intervals.

$\dagger$, Wilcoxon matched-pair signed ranks test; $₫$, Exact McNemar test for paired samples.

TABLE 2c: Ninety-five percent adherence to tuberculosis treatment before and after initiation of antiretroviral treatment.

\begin{tabular}{|c|c|c|c|c|c|c|c|c|}
\hline \multirow[t]{2}{*}{ Adherence measure } & \multicolumn{3}{|c|}{$\begin{array}{c}\text { Proportion of patients with } \geq 95 \% \\
\text { adherence pre ART }\end{array}$} & \multicolumn{3}{|c|}{$\begin{array}{c}\text { Proportion of patients with } \geq 95 \% \\
\text { adherence post ART }\end{array}$} & \multirow[t]{2}{*}{$\begin{array}{l}\text { Difference in proportion adherent } \\
\text { post v. pre cART }(\%)\end{array}$} & \multirow[t]{2}{*}{$p$ value } \\
\hline & $N$ & $\%$ & $95 \% \mathrm{Cl}$ & $N$ & $\%$ & $95 \% \mathrm{Cl}$ & & \\
\hline Pill count & 50 & 78.0 & $66.1-89.9$ & 0.36 & 68.0 & $54.6-81.4$ & -10.0 & 0.36 \\
\hline Electronic MEMS & 21 & 47.6 & $24.3-70.9$ & $1.00 \dagger$ & 30.0 & $8.0-52.0$ & -17.6 & $1.00 \dagger$ \\
\hline
\end{tabular}

MEMS, Medication Event Monitoring System; ART, antiretroviral treatment; $\mathrm{Cl}$, confidence intervals.

$\dagger$, Wilcoxon matched-pair signed ranks test; $₫$, Exact McNemar test for paired samples.

TABLE 2d: Ninety percent adherence to tuberculosis treatment before and after initiation of antiretroviral treatment.

\begin{tabular}{|c|c|c|c|c|c|c|c|c|}
\hline \multirow[t]{2}{*}{ Adherence measure } & \multicolumn{3}{|c|}{$\begin{array}{c}\text { Proportion of patients with } \geq 90 \% \\
\text { adherence pre ART }\end{array}$} & \multicolumn{3}{|c|}{$\begin{array}{c}\text { Proportion of patients with } \geq 90 \% \\
\text { adherence post ART }\end{array}$} & \multirow[t]{2}{*}{$\begin{array}{c}\text { Difference in proportion adherent } \\
\text { post v. pre cART }(\%)\end{array}$} & \multirow[t]{2}{*}{$p$ value: } \\
\hline & $N$ & $\%$ & $95 \% \mathrm{Cl}$ & $N$ & $\%$ & $95 \% \mathrm{Cl}$ & & \\
\hline Pill count & 50 & 88.0 & $78.7-97.3$ & 0.18 & 78.0 & $66.1-89.9$ & -10.0 & 0.18 \\
\hline Self-report & 50 & 100 & $100-100$ & 1.0 & 100 & $92.9-100$ & 0 & 1.0 \\
\hline Electronic MEMS & 21 & 61.9 & $39.3-84.6$ & $0.63 \dagger$ & 40.0 & $16.5-63.5$ & -21.9 & $0.63 \dagger$ \\
\hline
\end{tabular}

MEMS, Medication Event Monitoring System; ART, antiretroviral treatment; $\mathrm{Cl}$, confidence intervals

$\dagger$, Wilcoxon matched-pair signed ranks test; $\downarrow$, Exact McNemar test for paired samples.

for 21 participants pre ART, 20 participants post ART, and 14 both before and after ART initiation. The proportion of participants adherent to TB treatment after ART initiation was consistently lower than adherence before ART initiation: $10.0 \%(0.4-24.4)$ versus 38.1 (15.4-60.7); 30.0 (8.0-52.0) versus 47.6 (24.3-70.9) and 40.0 (16.5-63.5) versus $61.9(39.3-84.6)$ when adherence was defined as $100 \%, 95 \%$ or $90 \%$ of prescribed doses taken, respectively.

\section{Reasons for suboptimal adherence}

Leaving house without tablets $(n=8)$ and running out of tablets between visits $(n=8)$ were the most frequently stated reasons for missing doses $(n=8)$, followed by forgetfulness $(n=3)$, dosing errors $(n=3)$, taking medication as prescribed whilst not using the eMEMS lid $(n=3)$, distractions $(n=2)$, medication side-effects $(n=2)$ and lack of transport money $(n=1)$.

\section{Factors associated with optimal adherence to TB treatment after antiretroviral therapy initiation}

Employment status was the only factor associated with optimal (100\%) adherence following ART initiation. Compared with those not employed, participants who were employed had four times greater odds (aOR 4.11, 95\% CI 1.06-16.0) of being fully adherent to TB treatment. Age and gender tended to be associated with optimal adherence, with a $13 \%(95 \%$ CI $-1,+29 \%)$ increased odds for every year increase in age and men being less likely to optimally adhere to TB treatment (aOR 0.32, 95\% CI 0.08-1.29), but these associations did not reach statistical significance. In sensitivity analyses, age and employment status were factors associated with $95 \%$ and $90 \%$ adherence, respectively (Table 3).

\section{Discussion}

In the present study of ART-naïve HIV-infected individuals receiving treatment for active $\mathrm{TB}$, we observed a trend of decrease in adherence to TB treatment in the first month following ART initiation, with an $8 \%-10 \%$ decrease in the proportion of patients adherent according to pill count and an $18 \%-22 \%$ decrease in the proportion of patients adherent according to eMEMS, independent of the cut-off used to define adherence $(90 \%, 95 \%$ or $100 \%$ of prescribed doses taken). The finding of reduced adherence soon after the introduction of ART is clinically relevant, as suboptimal adherence has been associated with poor treatment 
TABLE 3: Factors associated with optimal adherence ( $100 \%$ of prescribed doses) following initiation of antiretroviral treatment in patients receiving treatment for active tuberculosis (pe 0.05; pr 0.15).

\begin{tabular}{|c|c|c|c|c|}
\hline Factor & Characteristic & Crude OR $(95 \% \mathrm{Cl})$ & $\begin{array}{l}\text { Adjusted OR full model } \\
(95 \% \mathrm{Cl})\end{array}$ & $\begin{array}{l}\text { Adjusted OR final model } \\
(95 \% \mathrm{Cl})\end{array}$ \\
\hline Age & Per year increase & $1.12(1.00-1.25)$ & $1.11(0.97-1.28)$ & $1.13(0.99-1.29)$ \\
\hline \multirow[t]{2}{*}{ Gender } & Female & Referent & Referent & Referent \\
\hline & Male & $0.80(0.26-2.45)$ & $0.34(0.60-1.95)$ & $0.32(0.08-1.29)$ \\
\hline \multirow[t]{2}{*}{ Nationality } & South African & Referent & Referent & - \\
\hline & Not South African & $0.83(0.27-2.55)$ & $1.48(0.30-7.14)$ & - \\
\hline \multirow[t]{2}{*}{ Education } & Secondary school not complete & Referent & Referent & - \\
\hline & Secondary school completed & $0.36(0.10-1.27)$ & $0.37(0.07-1.91)$ & - \\
\hline \multirow[t]{2}{*}{ Employment } & Unemployed & Referent & Referent & Referent \\
\hline & Employed & $4.53(1.30-15.77)$ & $3.87(0.80-18.8)$ & $4.11(1.06-16.0)$ \\
\hline CD4 count & (per 100 cells $/ \mathrm{mm}^{3}$ decrease) & $1.37(0.61-3.00)$ & $1.19(0.43-3.30)$ & - \\
\hline BMI & Per unit increase & $0.98(0.85-1.14)$ & $1.01(0.92-1.11)$ & - \\
\hline Time between TB treatment and ART initiation & Per day increase & $1.01(0.98-1.02)$ & $1.01(0.98-1.04)$ & - \\
\hline Adherence to TB treatment before ART initiation & $<100 \%$ & Referent & Referent & - \\
\hline \multirow[t]{2}{*}{ Family DOT post ART } & No & Referent & Referent & - \\
\hline & Yes & $0.40(0.12-1.25)$ & $0.81(0.16-4.06)$ & - \\
\hline
\end{tabular}

$\mathrm{BMI}$, body mass index; TB, tuberculosis; ART, antiretroviral treatment; DOT, directly observed treatment; OR, odds ratios; Cl, confidence intervals.

outcomes and development of resistance, especially in the early phases of treatment when the bacillary load is highest. Whilst many have speculated that the increased pill count in patients with TB initiating ART could reduce adherence, ${ }^{5,6,9}$ we could not compare our findings with others as we could not find published reports assessing this association.

Similar to findings of other studies, ${ }^{2}$ reasons for nonadherence reported by patients were multifactorial and few independent predictors for optimal adherence could be identified. Except for employment, with those being employed having four times higher odds of remaining fully adherent when initiating ART, we could not identify patient factors associated with adherence.

In the present study, we used three different methods to measure adherence: self-report of missed doses, pill count, and eMEMS. We observed that adherence by self-report was always highest, pill count gave intermediate estimates, and eMEMS consistently resulted in the lowest estimates. Poor correlations between different adherence measures have been reported. For example, in a study of adherence to ART, Holzemer found that there was minimal correlation amongst adherence as measured by pharmacy refill, selfreport, MEMS and pill count. ${ }^{10}$ Overestimation of adherence by self-report is a consistent finding, probably related to social desirability or recall error. ${ }^{11}$ eMEMS, on the other hand, can underestimate adherence when several doses of medications are removed from bottles at a single time, as was observed in the present and other studies. ${ }^{12,13}$ Similar to what was observed in our cohort, self-reported rates of adherence are higher than the rates derived from electronic monitoring; however, the $40 \%-50 \%$ difference between the two measures is greater than the $10 \%-30 \%$ reported in other settings. ${ }^{14,15}$ This result may be owing to limited validity of the eMEMS data, given the numerous challenges when implementing eMEMS into routine care in a resource-limited setting, including batteries of eMEMS caps running flat, power cuts during the transfer of eMEMS data to computer, errors made by pharmacists when filling the containers, and breakage of the container lids. In addition, some patients forgot or lost their Securitainer.

The present study has several limitations. Firstly, the small sample resulted in imprecise estimates and a lack of power to detect statistically significant differences, even when the differences observed were probably of clinical relevance. Secondly, the time between starting TB treatment and ART initiation in the study participants was short, (median of 27 days), and did not vary greatly between participants (IQR 13-48). As such, we could not assess the impact of timing of ART initiation on adherence. It is possible that delay of ART initiation until the end of the intensive phase, when patients feel better and side-effects of TB treatment have subsided, could lower the negative impact of ART initiation on adherence to TB treatment. This possibility would need to be weighed against the risk of poor treatment outcomes owing to the delay of ART initiation, and may therefore only be possible for patients with high CD4 counts at the time of TB diagnosis. Thirdly, we limited our assessment to the 28 days immediately before and after ART initiation to assess the impact of ART initiation on adherence to TB drugs. We could therefore not assess whether the observed changes in adherence were temporary or persisted throughout the TB treatment period. Finally, the clinic did not perform clinicbased directly observed treatment (DOT). Findings of the present study may therefore not be generalisable to settings where DOT is systematically implemented for all patients receiving TB treatment.

\section{Conclusion}

In the present small prospective cohort study, we observed a trend to decreased adherence to TB treatment following the initiation of ART. Our findings suggest that adherence 
interventions in the period following ART initiation may be needed to optimise treatment outcomes for people living with TB and HIV.

\section{Acknowledgements}

This work was supported by PEPFAR and the National Institutes of Health grant UM1 AI069463, the United States Agency for International Development grants to Right to Care: 674-A-00-08-00007 and 674-A-12-00020 and by the University of the Witwatersrand, Faculty of Health Sciences Research Committee. We are also thankful to Jacques de Vos (GeoMed) for providing the eMEMS and securitainers ${ }^{\circledast}$. We are grateful to the study participants, the staff at Witkoppen Health and Welfare Center (Bridgette Moatlhodi, Katerina Roussos), Rightmed Pharmacy (Coreen Barker) and the Medical Research Council (Prof Piet Becker).

\section{Competing interests}

The authors declare that they have no financial or personal relationships which may have inappropriately influenced them in writing this article.

\section{Authors' contributions}

M.K. (University of the Witwatersrand) collected the data and wrote the first draft. R.L.v.Z. (University of the Witwatersrand) was involved in project design, I.S. (Right to Care) made conceptual contributions, J.B. (Witkoppen Health and Welfare Centre) was involved in data collection, and A.v.R. (University of North Carolina) was involved in project design and statistical analysis. All authors reviewed the manuscript and gave scientific input.

\section{References}

1. World Health Organization. Adherence to long-term therapies - Evidence for action. Geneva: World Health Organization; 2003.

2. Munro SA, Lewin SA, Smith HJ, Engel ME, Fretheim A, Volmink J. Patient adherence to tuberculosis treatment: A systematic review of qualitative research. PLoS Med. 24 July 2007;4(7):e238. PMID: 17676945, http://dx.doi.org/10.1371/journal. pmed.0040238

3. World Health Organization. WHO policy in collaboratove TB/HIV activities: Guidelines for national programmes and other stakeholders. Geneva: World Health Organization; 2012.

4. Havlir DV, Kendall MA, Ive P, et al. Timing of antiretroviral therapy for HIV-1 infection and tuberculosis. N Engl J Med. 2011:365:1482-1491. PMID: 22010914, $\mathrm{http}: / / \mathrm{dx}$.doi.org/10.1056/NEJMoa1013607

5. Naidoo K, Baxter C, Abdool Karim SS. When to start antiretroviral therapy during tuberculosis treatment? Curr Opin Infect Dis. 2013;26:35-42. PMID: 23188213, http://dx.doi.org/10.1097/QCO.0b013e32835ba8f9

6. Torok ME, Farrar JJ. When to start antiretroviral therapy in HIV-associated tuberculosis. N Engl J Med. 2011;365:1538-1540. PMID: 22010921, http://dx.doi. org/10.1056/NEJMe1109546

7. World Health Organization. Global tuberculosis report. Geneva: World Health Organization; 2013.

8. Varma JK, Nateniyom S, Akksilp S, et al. HIV care and treatment factors associated with improved survival during TB treatment in Thailand: an observational study. BMC Infect Dis. 2009;9:42. PMID: 19364398, http://dx.doi.org/10.1186/1471 2334-9-42

9. Swaminathan S, Padmapriyadarsini C, Narendran G. HIV-associated tuberculosis: Clinical update. Clin Infect Dis. 2010;50:1377-1386. PMID: 20388036, http:// dx.doi.org/10.1086/652147

10. Holzemer WL, Bakken S, Portillo $\mathrm{CJ}$, et al. Testing a nurse-tailored HIV medication adherence intervention. Nurs Res. 2006:55:189-197. PMID: 16708043, http:// dx.doi.org/10.1097/00006199-200605000-00005

11. Wagner G, Miller LG. Is the influence of social desirability on patients' selfreported adherence overrated? J Acquir Immune Defic Syndr. 2004;35:203-204. PMID: 14722455, http://dx.doi.org/10.1097/00126334-200402010-00016

12. Ailinger RL, Black PL, Lima-Garcia N. Use of electronic monitoring in clinical nursing research. Clin Nurs Res. 2008;17:89-97. PMID: 18387881, http://dx.doi. org/10.1177/1054773808316941

13. Kalichman SC, Amaral CM, Cherry C, et al. Monitoring medication adherence by unannounced pill counts conducted by telephone: reliability and criterionrelated validity. HIV Clin Trials. 2008;9:298-308. PMID: 18977718, http://dx.doi. org/10.1310/hct0905-298

14. Thirumurthy $\mathrm{H}$, Siripong $\mathrm{N}$, Vreeman RC, et al. Differences between self-reported and electronically monitored adherence among patients receiving antiretrovira therapy in a resource-limited setting. AIDS. 2012;26:2399-2403. PMID: 22948266, http://dx.doi.org/10.1097/QAD.0b013e328359aa68

15. Liu H, Golin CE, Miller LG, et al. A comparison study of multiple measures of adherence to HIV protease inhibitors. Ann Intern Med. 2001;134:968-977. PMID: 11352698, http://dx.doi.org/10.7326/0003-4819-134-10-200105150-00011 